VoL. 71 (2005) [37-40]

\title{
ON EXTREMALITY OF TWO CONNECTED LOCALLY EXTREMAL BELTRAMI COEFFICIENTS
}

\begin{abstract}
GuOWU YaO
Let $\Omega_{1}$ and $\Omega_{2}$ be two domains in the complex plane with a nonempty intersection. Suppose that $\mu_{j}$ are locally extremal Beltrami coefficients in $\Omega_{j}(j=1,2)$ respectively. In 1980 , Sheretov posed the problem: Will the coefficient $\mu$ defined by the condition $\mu(z)=\mu_{j}(z)$ for $z \in \Omega_{j}, j=1,2$, be locally extremal in $\Omega_{1} \cup \Omega_{2}$ ? We give a counterexample to show that $\mu$ may not be locally extremal and not even be extremal.
\end{abstract}

\section{INTRODUCTION}

Let $\mathfrak{D}$ be a domain in the complex plane $\mathbb{C}$ with at least two boundary points and Let $M(\mathfrak{D})$ be the open unit ball of $L^{\infty}(\mathfrak{D})$. Every element $\mu \in M(\mathfrak{D})$ can be regarded as an element in $L^{\infty}(\mathbb{C})$ by putting $\mu$ equal to zero in the outside of $\mathfrak{D}$. Every $\mu \in M(\mathfrak{D})$ induces a global quasiconformal self-mapping $f$ of the plane which solves the Beltrami equation [1],

$$
f_{\bar{z}}(z)=\mu(z) f_{z}(z),
$$

and $f$ is defined uniquely up to postcomposition by a complex affine map of the plane. Conversely, any quasiconformal mapping $f$ defined on $\mathfrak{D}$ has a Beltrami coefficient $\mu(z)=f_{\bar{z}}(z) / f_{z}(z)$ in $M(\mathfrak{D})$.

Two Beltrami coefficients $\mu, \nu \in M(\mathfrak{D})$ are equivalent if they induce quasiconformal mappings $f$ and $g$ by (1) such that there is a conformal map $c$ from $f(\mathfrak{D})$ to $g(\mathfrak{D})$ and an isotopy through quasiconformal mappings $h_{t}, 0 \leqslant t \leqslant 1$, from $\mathfrak{D}$ to $\mathfrak{D}$ which extend continuously to the boundary of $\mathfrak{D}$ such that

1. $h_{0}(z)$ is identically equal to $z$ on $\mathfrak{D}$,

2. $h_{1}$ is identically to $g^{-1} \circ c \circ f$, and

3. $h_{t}(p)=g^{-1} \circ c \circ f(p)$ for any $p \in \partial \mathfrak{D}$.

Received 22nd July, 2004

The author was supported by China Postdoctoral Science Foundation and by the National Natural Science Foundation of China (Grant No. 10401036).

Copyright Clearance Centre, Inc. Serial-fee code: 0004-9727/05 \$A2.00+0.00. 
The equivalence relation partitions $M(\mathfrak{D})$ into equivalence classes and the space of equivalence classes is by definition the Teichmüller space $T(\mathfrak{D})$ of $\mathfrak{D}$.

Given $\mu \in M(\mathfrak{D})$, we denote by $[\mu]$ the set of all elements $\nu \in M(\mathfrak{D})$ equivalent to $\mu$, and set

$$
k_{0}([\mu])=\inf \left\{\|\nu\|_{\infty}: \nu \in[\mu]\right\} .
$$

We say that $\mu$ is extremal (in $[\mu])$ if $\|\mu\|_{\infty}=k_{0}([\mu]), \mu$ is uniquely extremal if $\|\nu\|_{\infty}$ $>k_{0}([\mu])$ for any other $\nu \in[\mu]$; the alternative is that $\mu$ is non-uniquely extremal.

We define $A(\mathfrak{D})$ as the Banach space of all holomorphic functions $\varphi$ on $\mathfrak{D}$ with $L^{1}-$ norm

$$
\|\varphi\|=\iint_{\mathfrak{D}}|\varphi(z)|<\infty
$$

As is well known, a necessary and sufficient condition (Hamilton-Krushkal-ReichStrebel condition) that a Beltrami coefficient $\mu$ is extremal in its class in $T(\mathfrak{D})$ is that [4] it has a so-called Hamilton sequence, namely, a sequence $\left\{\phi_{n} \in A(\mathfrak{D}):\left\|\phi_{n}\right\|=1, n \in \mathbb{N}\right\}$, such that

$$
\lim _{n \rightarrow \infty} \iint_{\mathfrak{D}} \mu \phi_{n}(z) d x d y=\|\mu\|_{\infty} .
$$

A Beltraim coefficient $\mu$ in $\mathfrak{D}$ is called to be locally extremal if for any domain $G \subset \mathfrak{D}$ it is extremal in its class in $T(G)$; in other words,

$$
\|\mu\|_{G}:=\operatorname{essup}_{z \in G}|\mu|=\sup \left\{\frac{\left|\iint_{G} \mu \phi_{n}(z) d x d y\right|}{\|\phi\|}: \phi \in A(G)\right\} .
$$

Obviously, extremality in the whole domain is a prerequisite for a Beltrami coefficient to be locally extremal.

In [6], Sheretov investigated locally extremal Beltrami coefficients and posed the following problem: Let $\Omega_{1}$ and $\Omega_{2}$ be two domains with $\Omega_{1} \cap \Omega_{2} \neq \emptyset$. Suppose that $\mu_{j}$ are locally extremal Beltrami coefficients in $\Omega_{j}(j=1,2)$ respectively. Will the coefficient $\mu$ defined by the condition $\mu(z)=\mu_{j}(z)$ for $z \in \Omega_{j}, j=1,2$, be locally extremal in $\Omega_{1} \cup \Omega_{2}$ ?

The main purpose of this paper is to give a negative answer to the above problem in a stronger sense. We shall construct certain counterexample in the next section.

\section{Construction of COUNTEREXAMPLE}

If $\mu$ in $M(\mathfrak{D})$ is uniquely extremal in its class $[\mu]$ in $T(\mathfrak{D})$, then it is obviously locally extremal. But the converse is not true for which here we include the example constructed in [2, Theorem 2.2] by Reich.

Reich's example: We denote the parabolic region $\Omega_{0}$ by

$$
\Omega_{0}=\left\{z=x+i y: x>y^{2}, x>0\right\} .
$$


In $\Omega_{0}$, we define $\mu(z) \equiv k$ where $k \in(0,1)$ is a constant. Examining the proof of $[2$, Theorem 2.2], we find that

$$
\sup \left\{\frac{\left|\iint_{G} \mu(z) \phi(z) d x d y\right|}{\iint_{G} \mid \phi \| d x d y}: \phi(z) \in A\left(\Omega_{0}\right)\right\}=k
$$

for any positive measure subset $G$ of $\Omega_{0}$. This relation indicates that $\mu$ is locally extremal in $\Omega_{0}$. But, it is well known that $\mu$ is not uniquely extremal (see $[2,3]$ ).

In our counterexample to Sheretov's problem, $\mu_{j}, j=1,2$, are uniquely extremal while $\mu$ may not be locally extremal and not even be extremal in its corresponding class. EXAmple 1. Let $\Delta$ be the unit disk $\{z:|z|<1\}$. Put

$$
\Omega_{1}=\left\{z \in \Delta: \arg z \in\left(-\frac{\pi}{2}, \frac{\pi}{2}\right)\right\}, \Omega_{2}=\left\{z \in \Delta:|\arg z|>\frac{\pi}{4}\right\} .
$$

Obviously, $\Omega_{1} \cup \Omega_{2}=\Delta^{*}=\Delta-\{0\}$ and $\Omega_{1} \cap \Omega_{2} \neq \emptyset$. Set $\mu=k \bar{\varphi} / \mid \varphi$ on $\Delta$, where $k \in(0,1)$ is a constant and $\varphi(z)=1 / z^{2}$. Let $\mu_{j}(j=1,2)$ be the restrictions of $\mu$ on $\Omega_{j}$, respectively. We claim that $\mu_{j}$ are uniquely extremal in their classes in $T\left(\Omega_{j}\right)$, respectively.

Suppose the conformal mapping $z=F(\zeta)$ maps $\Delta_{\zeta}=\{|\zeta|<1\}$ onto $\Omega_{1}$. The question becomes that of determining whether the Beltrami coefficient

$$
\widetilde{\mu}=k \frac{\overline{\varphi \circ F}}{|\varphi \circ F|} \frac{\overline{F^{\prime}(\zeta)^{2}}}{\left|F^{\prime}(\zeta)\right|^{2}}
$$

is extremal or uniquely extremal in its class in $T\left(\Delta_{\zeta}\right)$. Set

$$
\psi(\zeta)=(\varphi \circ F) F^{\prime}(\zeta)^{2}
$$

Because the conformal mapping $F^{-1}$ transfers the second order pole of $\varphi(z)$ to the second order pole of $\psi(\zeta)$, it is not difficult to see that $\psi(\zeta)$ is holomorphic in $\Delta_{\zeta}$ and is meromorphic in $\overline{\Delta_{\zeta}}$ except that it has a pole of second order at $\zeta=F^{-1}(0)$. Thus, by [5, Theorem 6], $\tilde{\mu}$ is uniquely extremal in its class in $T\left(\Delta_{\zeta}\right)$, and hence $\mu_{1}$ is uniquely extremal in its class in $T\left(\Omega_{1}\right)$. Similarly, $\mu_{2}$ is uniquely extremal in its class in $T\left(\Omega_{2}\right)$.

However, $\mu$ is not even extremal in $[\mu]$ in $T\left(\Delta^{*}\right)$. In fact, noting that $\left\{z^{n}\right.$ : $n=-1,0,1,2, \ldots\}$ is a base of the Banach space $A\left(\Delta^{*}\right)$ and

$$
\iint_{\Delta^{*}} \mu(z) \phi(z) d x d y=\iint_{\Delta^{*}} k \frac{z^{2}}{|z|^{2}} z^{n} d x d y=0, n=-1,0,1,2, \ldots,
$$

it follows readily that

$$
\sup \left\{\left|\iint_{\Delta^{*}} \mu(z) \phi(z) d x d y\right| /\|\phi\|: \phi(z) \in A\left(\Delta^{*}\right)\right\}=0 .
$$

Thus, $\mu$ is not extremal in its class in $T\left(\Delta^{*}\right)$ by the condition of Hamilton sequence. And hence, $\mu$ is not locally extremal in $\Delta^{*}$. 
Notice that in the above example, $\Omega_{1} \cap \Omega_{2}$ contains two connected components. If the condition $\Omega_{1} \cap \Omega_{2} \neq \emptyset$ in the original problem replaced by that $\Omega_{1} \cap \Omega_{2}$ is connected, what situation should be? Up to the present, we can not find such a counterexample.

REMARK 1. After the completion of this paper I have become aware of a paper with related result: Zhong Li et al., An extremal problem of quasiconformal maps, to appear in Proc. Amer. Math. Soc.

\section{REFERENCES}

[1] L.V. Ahlfors and L. Bers, 'Riemann's mapping theorem for variable metrics', Ann. Math. 72 (1960), 385-404.

[2] E. Reich, 'On the uniqueness question for Hahn-Banach extensions from the space of $L^{1}$ analytic functions', Proc. Amer. Math. Soc. 88 (1983), 305-310.

[3] E. Reich and K. Strebel, 'On the extremality of certain Teichmüller mappings', Comment. Math. Helv. 45 (1970), 353-362.

[4] E. Reich and K. Strebel, 'Extremal quasiconformal mappings with given boundary values', in Contributions to Analysis, A Collection of Papers Dedicated to Lipman Bers (Academic Press, New York, 1974), pp. 375-392.

[5] G.C. Sethares, 'The extremal property of certain Teichmüller mappings', Comment. Math. Helv. 43 (1968), 98-119.

[6] V.G. Sheretov, 'Locally extremal quasiconformal mappings', Soviet Math. Dokl. 21 (1980), 343-345.

Institute of Mathematics

Academy of Mathematics and Systems Science

Chinese Academy of Sciences

Beijing 100080

People's Republic of China

e-mail: wallgreat@lycos.com 